\title{
Identification of chemical constituents from fruit of Antidesma bunius by GC-MS and HPLC-DAD-ESI-MS
}

\author{
Yelliantty YELLIANTTY ${ }^{1}$ (D), Rahmana Emran KARTASASMITA, Slamet Ibrahim SURANTAATMADJA ${ }^{1}$, \\ Yaya RUKAYADI ${ }^{2}$
}

\begin{abstract}
Antidesma bunius is an edible berry fruit with many benefits, such as natural antimicrobials, anticancer, natural dyes, etc. However, data on chemical content in the fruit is still limited. The purpose of this research is to identify volatile compounds of Antidesma bunius fruit. We extracted and analyzed the A. bunius fruit's chemical content using GC-MS and HPLC-DAD-ESI-MS methods. Forty-nine compounds representing $99.91 \%$ of the total chromatogram's relative peak area were detected. Antidesma bunius is rich in 5-hydroxymethylfurfural (HMF) and ten other compounds with relative peak area $>1 \%$, such as furaldehyde, citric acid and others. We also found 109 compounds tentatively identified through HPLC-DAD-ESI-MS. Antidesma bunius contained HMF, several volatile compounds, organic acid, long-chain fatty acid, and photochromic compound.
\end{abstract}

Keywords: Antidesma bunius; berry; bignay, GC-MS; HPLC-DAD-ESI -MS; volatile.

Practical Application: The study results indicate the possible use of the fruit of A. bunius for food flavoring, antimicrobial and anticancer agents.

\section{Introduction}

Antidesma bunius belongs to the Phyllantaceae family. The fruit is included in the type of berries. Antidesma bunius comes from east and south Asia. This plant has several other names, such as bignay, bignai, bugnay, Chinese laurel, Queensland cherry, wild cherry, currant tree, salamander-tree. In Indonesia, this fruit is known as the Buni or Huni fruit (Lizardo et al., 2015).

In Indonesia, Buni fruit is usually consumed directly when ripe or processed into local foods such as Rujak. The acidic taste and the striking color become an attraction for consumption. Besides its refreshing taste, Buni fruit is known to have many health benefits and can be applied to other aspects.

Antidesma bunius has many benefits for human health. Almost all parts of Antidesma bunius have bioactive compounds that can be used as a source of medicine. Buni fruit contains antioxidant compounds, anticancer, anti-inflammation, antidiabetic, anti-hyperlipidemia and can also be used as an antiparasitic agent (Chowtivannakul et al., 2016; Jorjong et al., 2015; Lizardo et al., 2015).

Health benefits and also applications of Buni fruit have been widely studied. However, data regarding the content of chemical compounds in the fruit are still limited. The research that has been carried out only covers the characterization of phytochemical content, polyphenol compounds and anthocyanin, but its chemical constituents remain incompletely investigated. Furthermore, no studies have examined the content of volatile compounds and essential oils in the fruit of Antidesma bunius. (Hardinasinta et al., 2020; Jorjong et al., 2015).

Thus, comprehensive profiling of the phytochemicals of $A$. bunius fruit using sensitive tools is necessary. Therefore, this study aims to analyze volatile compounds in the fruit of Antidesma bunius by using high-performance liquid chromatography-diode array detector-hyphenated with tandem mass spectrometry (HPLC-DAD-ESI-MS) and gas chromatography with tandem mass spectrometry (GC-MS) as a powerful analytical technique.

\section{Methods}

\subsection{Plant materials}

Fresh ripe fruit of Antidesma bunius was manually harvested in Sumedang, West Java Province, Indonesia during March 2019. The fruit was maintained at $-18^{\circ} \mathrm{C}$ immediately after collection until they were used.

\subsection{Extraction}

The fresh ripe fruit of Antidesma bunius (200 g) was crushed and then extracted using $1 \mathrm{~L}$ of $80 \%$ methanol in the darkroom temperature overnight. The extraction process was repeated three times to ensure exhaustive extraction. The supernatant is then filtered using filter paper, and the resulting filtrate is evaporated using a rotary evaporator at a temperature of $45^{\circ} \mathrm{C}$. 


\subsection{Gas Chromatography-Mass Spectrometry}

GCMS analyses were performed on a GC-2010 GC coupled with a GCMS-QP2010 Ultra mass spectrometer. The column was a Rxi-5ms fused-silica capillary column (30.0 m Length $\mathrm{x}$ $0.25 \mathrm{~mm}$ ID x $0.25 \mu \mathrm{m}$ Thickness) with helium as the carrier gas and was run at a constant pressure of $37.1 \mathrm{psi}$. The injection was conducted using a split-less mode at an injector temperature of $250{ }^{\circ} \mathrm{C}$. The oven temperature was ramped from 50 to $300{ }^{\circ} \mathrm{C}$ (10 min hold) at a rate of $3{ }^{\circ} \mathrm{C} / \mathrm{min}$. The oven temperature was held at $300{ }^{\circ} \mathrm{C}$ for $6 \mathrm{~min}$ following each analysis. The total run time for each sample was approximately $25 \mathrm{~min}$. The GCMS interface temperature was set to $250^{\circ} \mathrm{C}$. MS mode was used during analytical scanning from 40-700 atomic mass units (amu). The ion source temperature was set to $200{ }^{\circ} \mathrm{C}$. The blank was injected first, followed by the sample injection. The chromatograms obtained from the total ion current (TIC) were integrated without any correction for co-eluting peaks, and the results were expressed as total abundance. TIC peaks and chromatograms were analyzed using Agilent MSD ChemStation G1701DA software (version D 02.00, CA, USA). All peaks were identified based on mass spectral matching $(\geq 90 \%)$ from both the National Institute of Standards and Technology (NIST) and Wiley libraries. Only compounds with $90 \%$ or greater spectral matching accuracy were reported.

\subsection{HPLC-DAD-ESI-MS analysis}

Separation of phenolic compounds from Antidesma bunius extract was performed on an Agilent 1200 series Rapid Resolution LC (Agilent Technologies, Santa Clara, CA). This instrument was equipped with an Agilent Zorbax C18 column $(4.6 \times 150 \mathrm{~mm}, 5 \mu \mathrm{m})$ from Agilent Technologies. Acidified water $(0.5 \%$ acetic acid, $\mathrm{v} / \mathrm{v})$ and acetonitrile were used as mobile phases $\mathrm{A}$ and $\mathrm{B}$, respectively. The gradient was programmed as follows: 0 min, $0 \%$ B; 20 min, $20 \%$ B; 30 min, 30\% B; 40 min, 50\% B; $50 \mathrm{~min}, 75 \% \mathrm{~B} ; 60 \mathrm{~min}, 100 \% \mathrm{~B} ; 62 \mathrm{~min} 0 \% \mathrm{~B}$, and finally, the initial conditions were held for $8 \mathrm{~min}$ as a re-equilibration step. The flow rate was set at $0.80 \mathrm{~mL} / \mathrm{min}$ throughout the gradient.
The flow from the HPLC system into the ESI-MS detector was $0.2 \mathrm{~mL} / \mathrm{min}$. The injection volume was $10 \mu \mathrm{L}$ and the column temperature was maintained at $25^{\circ} \mathrm{C}$.

\section{Results and discussion}

\subsection{Chemical components identified in Antidesma bunius fruit by GC-MS}

A methanol extract of Antidesma bunius has a deep purple color. In the analysis of volatile components present in Antidesma bunius extract, a total of fifty compounds were detected (Figure 1). Among these components, 5-hydroxymethylfurfural (5-HMF) was found in the most significant concentrations, representing $47.07 \%$ of all volatiles. Other predominant components were furfural, citric acid, furan carboxylic acid, 2,3-dihydro-3,5-dihydroxy-6methyl-(4H)-pyran-4-one, hexanoic acid, 1,6-Anhydro-.beta.D-glucofuranose, 5,5'-oxy-Dimethylene-bis(2-Furaldehyde), Linolenic acid and gamma-Sitosterol.

The dominant compound detected in Antidesma bunius extract was 5-HMF with a relative amount of $47.07 \%$ (Table 1). This compound can be found naturally in honey and processed foods such as fruit juice, ketchup, UHT milk, etc. (Saeed et al., 2018). HMF is a compound that can be used as an intermediate for polymers, pharmaceuticals, liquid fuels, and the synthesis of several groups of compounds such as dialdehyde, ethers and other organic compounds. 5-HMF can be produced from hexoses such as fructose through several treatments such as dehydration (Gomes et al., 2015). This study result shows the potential of the Antidesma bunius fruit as a natural source of HMF.

This study also detected 5-HMF derivative compounds, namely 5.5'-oxy-Dimethylene-bis (2-Furaldehyde). This compound is the result of the thermal decomposition of 5-HMF. Formation of 5,5'-oxy-Dimethylene-bis (2-Furaldehyde) compounds occur at temperatures of $100-220^{\circ} \mathrm{C}$ (Nikolov \& Yaylayan, 2011).

One of predominant component detected in Antidesma bunius extract was furfural. This compound is commonly found in all fruits and vegetables and other plant parts that contain lignin

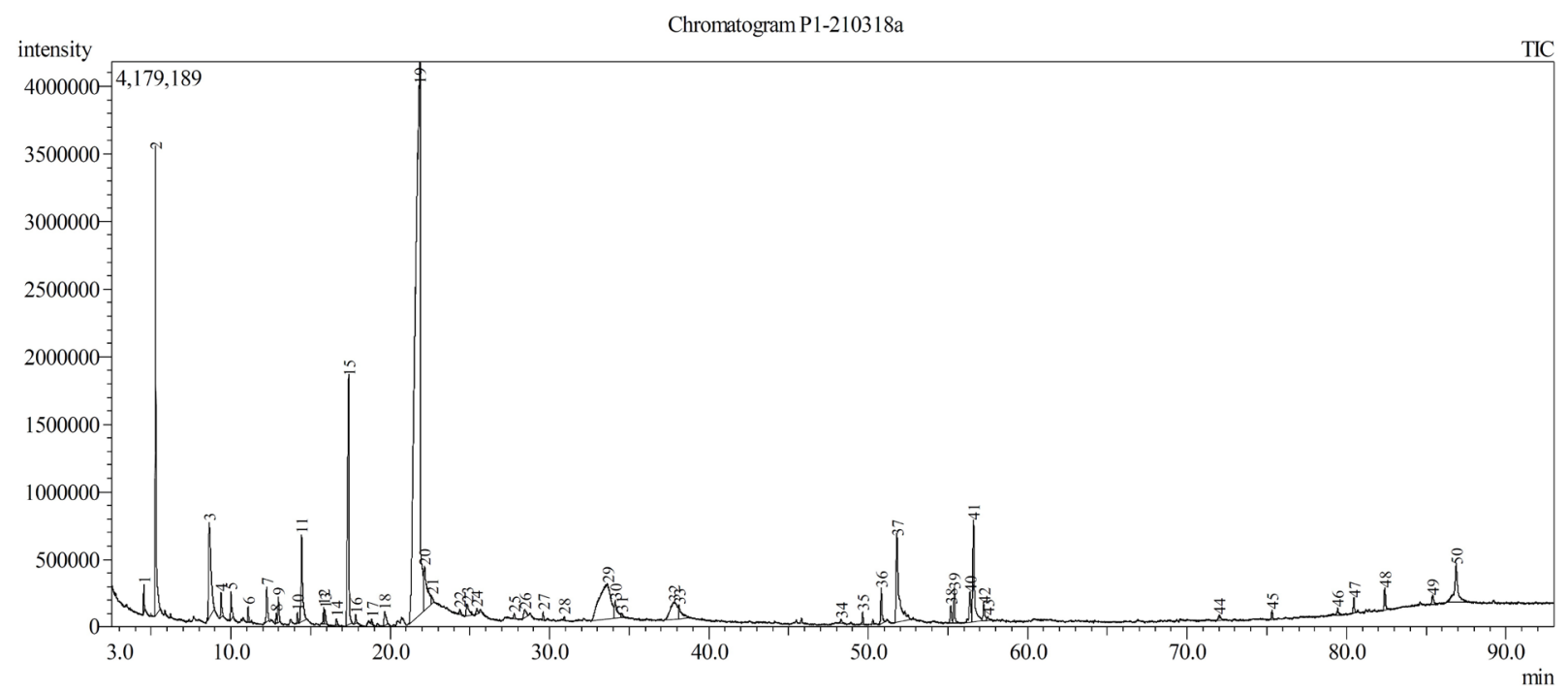

Figure 1. Gas Chromatogram of Volatile Compounds of Antidesma bunius extract. 
Table 1. Volatile components identified in Antidesma bunius fruit by GC-MS.

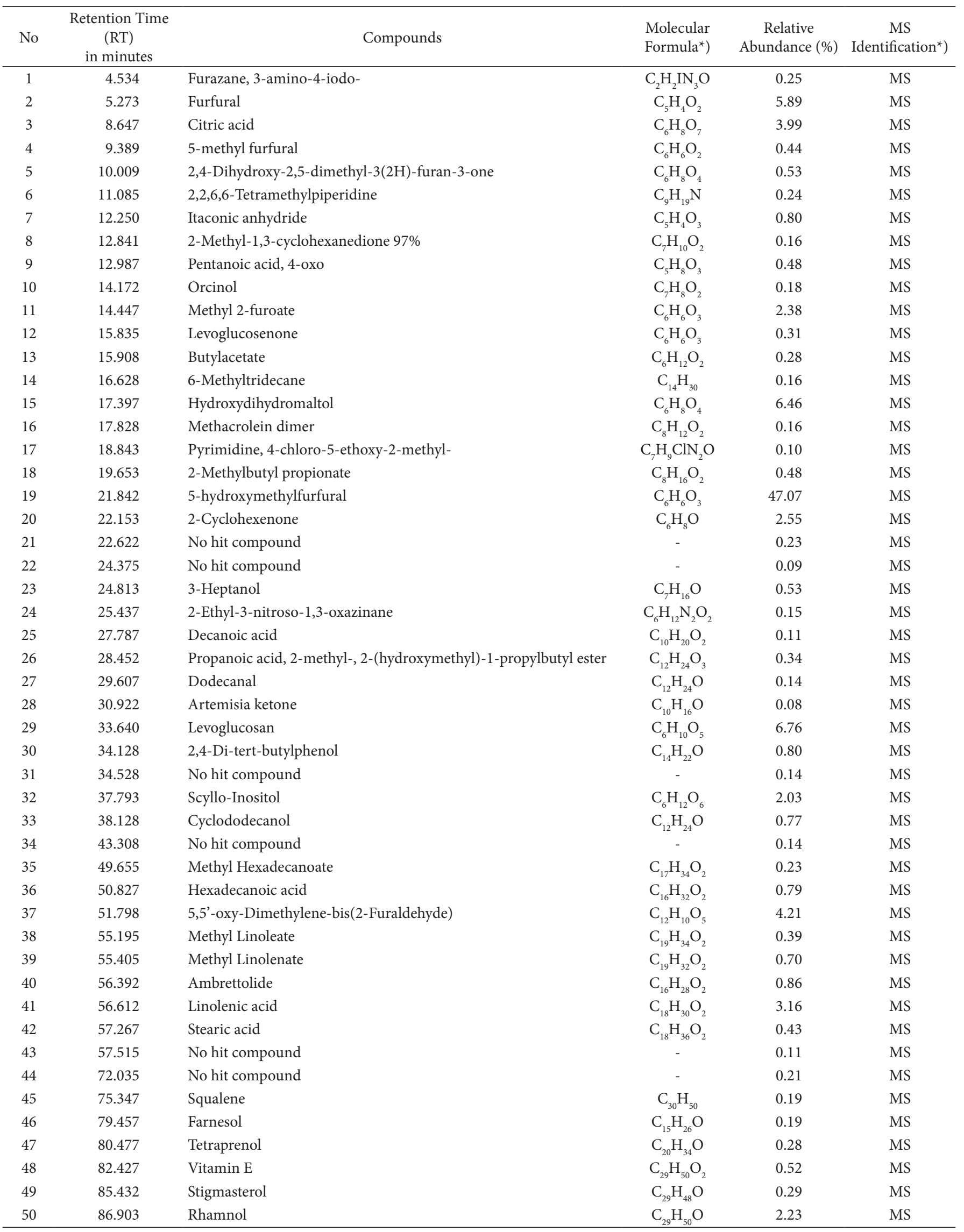

*) Based on comparison of MS spectrum with database of WILEY229.LIB. 
and cellulose. Furfural is also a multi-purpose compound such as 5-HMF. Furfural can be applied in the petroleum industry, medicine, and also agriculture as insecticides, pesticides, antiseptics, and can also be used as bio-fuel (Wankasi \& Tarawou, 2013).

Citric acid was also detected in Antidesma bunius extract (3.99\%). This compound is generally found in citrus fruits. Citric acid provides an acidic taste in some fruits such as lemon, orange, lime, grapefruit. Antidesma bunius fruit also has a strong sour taste. Based on this study results, citric acid may also be a contributor to Antidesma bunius fruit sour taste (Chanukya et al., 2017).

Furancarboxylic acid or 2 -furoic acid is a heterocyclic carboxylic acid, consisting of a five-membered aromatic ring and a carboxylic acid group. In the industry, this compound is used as a preservative, acting as a bactericide and fungicide. Antidesma bunius also own this antimicrobial property. Previous research shows the presence of antibacterial and antifungal activity from Antidesma bunius extract and food preservative effects (Lizardo et al., 2015). This study shows that 2 -furoic acid compounds can provide antimicrobial effects in Antidesma bunius.

Farnesol is a bioactive compound that is also detected in this study. Farnesol is a natural 15-carbon organic compound which is acyclic sesquiterpene alcohol. Farnesol is present in many essential oils and used in perfumery. Farnesol is also known as a natural pesticide for mites and flavoring ingredients. Farnesol has antibacterial activity and is used as a deodorant in cosmetic products. It has been reported to exhibit anticancer and anti-inflammatory effects and alleviate allergic asthma, gliosis, and edema. These findings support the development of Antidesma bunius as a potential source of the pharmacological agent (Jung et al., 2018; Kromidas et al., 2006).

Another compound detected in this study is Hydroxydihydromaltol (2,3-dihydro-3,5-dihydroxy-6-methyl-(4H)-pyran-4-one). It was identified as a novel potent aroma compound in a dairy product, such as Ryazhenka kefir, and found as odorants in roasted chicory (Preininger et al., 2008). This compound is thought to be formed due to the heating process and can affect processed foods' antioxidant activity. For example, increased Hydroxydihydromaltol is accompanied by an increase in antioxidant activity in dry prune products (Čechovská et al., 2011).

Hexanoic acid is an organic acid compound found in Antidesma bunius. This compound is also found in other fruit such as noni, pineapple and raspberry (Ferlinahayati et al., 2016; Aprea et al., 2015). Hexanoic acid has anticancer activity and also can improve energy metabolism (Narayanan et al., 2015; Miyamoto et al., 2016). Other studies have shown the anticancer activity of Antidesma bunius extract in Hela cells (Puspitasari \& Ulfa, 2009). Hexanoic acid can be one of the compounds that provide anticancer activity from Antidesma bunius.

Another predominant compound detected in Antidesma bunius fruit is 1,6-Anhydro-beta-D-glucofuranose. This compound is anhydrosugar and commonly formed from the results of glucose pyrolysis (Hu et al., 2017). However, this compound is also naturally found in Oak bark, Acacia honey and Punica granatum. (Deryabin \& Tolmacheva, 2015; Sangeetha \& Vijayalakshmi, 2011).
Linolenic acid is an essential fatty acid belonging to the omega-3 fatty acids group. It is highly concentrated in certain plant oils and fruits (Saini \& Keum, 2017). Linolenic acid has been reported to inhibit prostaglandin synthesis, resulting in reduced inflammation and the prevention of certain chronic diseases. It also has anticarcinogenic, lipid metabolism regulation, anti-inflammatory, anti-obese and antioxidant activities (Yuan et al., 2014).

Gamma-Sitosterol is a phytosterol with structural similarity to cholesterol and can be found in plants, animals, and fungi. This compound has many beneficial effects on human health, such as hypolipidemic agents, anticancer, antioxidant, etc. (Balamurugan et al., 2015). In this study, we detected gammasitosterol in Antidesma bunius. This result suggests that Antidesma bunius can be a hypolipidemic agent, as proven in a previous study (Chowtivannakul et al., 2016).

2,4-di-tert-butylphenol is a member of the class of phenols carrying two tert-butyl substituents at positions 2 and 4 . It has a role as a bacterial metabolite, an antioxidant, and a marine metabolite. It is an alkylbenzene and a member of phenols. Antioxidants/ Stabilizers Fuels and fuel additives Intermediate Processing aids.

This study also detected several compounds that can act as flavoring agents. 2-cyclohexenone has an organoleptic property such as roasted savory green odor, 2-Methylbutyl propionate, and Methyl 2-furoate taste like sweet caramel brown sugar musty.

\subsection{Chemical components identified in Antidesma bunius fruit by HPLC-DAD-ESI-MS}

Among those identified compounds, several compounds have not been detected in Antidesma bunius fruit before. This study is the first to describe these results. Table 2 shows the list of 109 compounds detected through HPLC-DAD-ESI-MS experiments along with their retention times (tR). Seventyeight compounds are tentatively identified, and 31 compounds remain unknown (Figure 2). The compounds detected in this work were tentatively characterized by MS data, together with the interpretation of the observed MS/MS spectra compared with those found in the literature. Several public databases were consulted in the identification process, such as ChemSpider (Royal Society of Chemistry, 2020), and PubChem (National Center for Biotechnology Information, 2020).

5-Methoxysalicylic acid is also known as 2-hydroxy-5methoxybenzoate. It is an extremely weak basic (nearly neutral) compound (based on its pKa). 5-Methoxysalicylic acid has been detected in herbs, spices and tea. (Dieryckx et al., 2015) However, this study results indicate that 5-Methoxysalicylic acid is also found in fruits such as A. bunius.

In this research, several fatty acid compounds were identified. (15Z)-9,12,13-Trihydroxy-15-octadecenoic acid is a long-chain fatty acid. This compound can be used for various purposes, such as antifoaming agents, coagulating agents, dispersion agents, emulsifiers, flotation agents, foaming agents, viscosity adjustors, etc. Butanedioic acid, 2-(6-hydroxyhexyl)-3-methylene- is a small acid found in Aspergillus tubingensis as its secondary metabolite. This compound has the potential to have bioactivity (Frisvad et al., 2018). However, there was no research to prove this. 


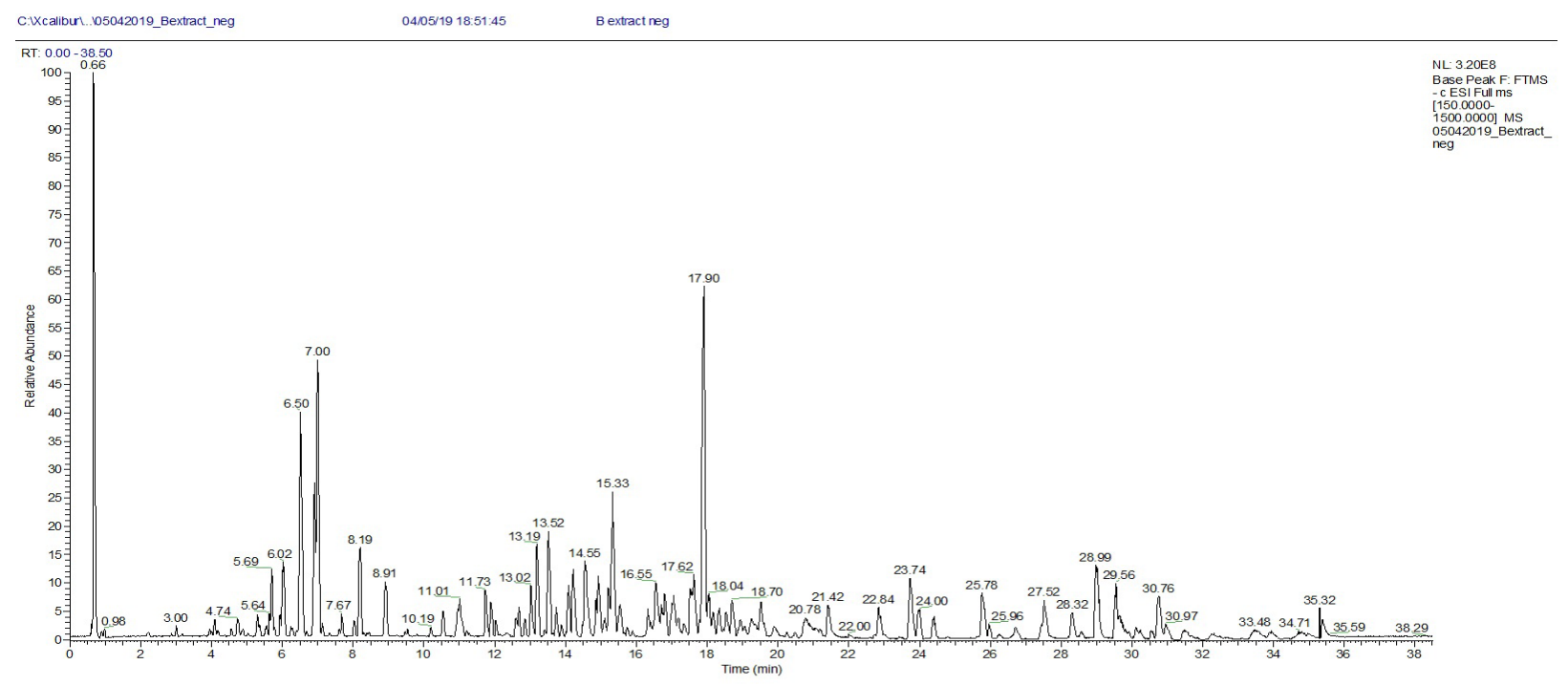

Figure 2. HPLC-DAD Base Peak Chromatogram (BPC) for Antidesma bunius extract.

Table 2. Chemical components identified in Antidesma bunius fruit by LC-MS/MS.

\begin{tabular}{|c|c|c|c|c|c|}
\hline $\begin{array}{l}\text { Peak } \\
\text { No }\end{array}$ & $\begin{array}{c}\text { Retention } \\
\text { Time (RT) in } \\
\text { minutes } \\
\end{array}$ & Compounds & $\begin{array}{l}\text { Molecular } \\
\left.\text { Weight }^{* *}\right)\end{array}$ & $\begin{array}{l}\text { Molecular } \\
\left.\text { Formula }^{*}\right)\end{array}$ & $\begin{array}{c}\text { Reference } \\
\quad \text { MS } \\
\text { Identification }^{\star *} \text { ) } \\
\end{array}$ \\
\hline 1 & 4.74 & 5-Methoxy salicylic acid & 168.041 & $\mathrm{C}_{8} \mathrm{H}_{8} \mathrm{O}_{4}$ & MS \\
\hline 2 & 5.3 & Suberic acid & 174.088 & $\mathrm{C}_{8} \mathrm{H}_{14} \mathrm{O}_{4}$ & MS \\
\hline 3 & 5.64 & $\begin{array}{l}\text { Butanedioic acid, 2-(6-hydroxyhexyl)-3-methylene- } \\
\text { Methyl 5-[2-(4-methoxy-2-methyl-4- }\end{array}$ & 230.115 & $\mathrm{C}_{11} \mathrm{H}_{18} \mathrm{O}_{5}$ & MS \\
\hline 4 & 5.68 & $\begin{array}{l}\text { oxobutyl)-5-methyl-1,3-dioxolan-4- } \\
\text { yl]pentanoate }\end{array}$ & 316.188 & $\mathrm{C}_{16} \mathrm{H}_{28} \mathrm{O}_{6}$ & MS \\
\hline 5 & 5.69 & Lariciresinol 4-O-glucoside & 568.215 & $\mathrm{C}_{26} \mathrm{H}_{34} \mathrm{O}_{11}$ & MS \\
\hline 6 & 5.94 & Unknown & 497.253 & $\mathrm{C}_{28} \mathrm{H}_{31} \mathrm{~N}_{7} \mathrm{O}_{2}$ & MS \\
\hline 7 & 6.02 & $\begin{array}{l}\text { Similar to: 1-(Carboxymethyl)cyclohexanecarboxylic } \\
\text { Acid; } \Delta \text { Mass: -208.0708 Da) }\end{array}$ & 394.16 & $\mathrm{C}_{16} \mathrm{H}_{22} \mathrm{~N}_{6} \mathrm{O}_{6}$ & MS \\
\hline 8 & 6.03 & $\begin{array}{l}\text { 1- (Carboxymethyl)cyclohexanecarboxylic } \\
\text { Acid }\end{array}$ & 186.088 & $\mathrm{C}_{9} \mathrm{H}_{14} \mathrm{O}_{4}$ & MS \\
\hline 9 & 6.51 & Phospocholine conjugate acid & 184.073 & $\mathrm{C}_{5} \mathrm{H}_{15} \mathrm{NO}_{4} \mathrm{P}$ & MS \\
\hline 10 & 6.51 & Similar to: Disperse red 17 & 390.129 & $\mathrm{C}_{17} \mathrm{H}_{20} \mathrm{~N}_{4} \mathrm{O}_{4}$ & MS \\
\hline 11 & 6.51 & Unknown & 590.167 & $\mathrm{C}_{28} \mathrm{H}_{27} \mathrm{~N}_{6} \mathrm{O}_{7} \mathrm{P}$ & - \\
\hline 12 & 6.51 & $\begin{array}{l}\text { Unknown } \\
\text { [Similar to: 2-(3,4- } \\
\text { Dihydroxyphenyl)ethyl (1S,4aR,7aR)-1- }\end{array}$ & 796.223 & $\mathrm{C}_{26} \mathrm{H}_{43} \mathrm{~N}_{10} \mathrm{O}_{13} \mathrm{P}_{3}$ & - \\
\hline 13 & 6.51 & $\begin{array}{l}\text { ( } \beta \text {-D-glucopyranosyloxy)-4a-hydroxy-7- } \\
\text { methyl-5-oxo-1,4a,5,6,7,7ahexahydrocyclopenta[c]pyran-4- } \\
\text { carboxylate; } \Delta \text { Mass: -68.0201 Da] }\end{array}$ & 594.189 & $\mathrm{C}_{21} \mathrm{H}_{37} \mathrm{~N}_{6} \mathrm{O}_{8} \mathrm{P}_{3}$ & MS \\
\hline 14 & 6.51 & $\begin{array}{l}\text { Malonic acid,isobutylidene-,bimol. cyclic ethylene ester } \\
\text { [Similar to: } 2-\left(3,4^{-}\right.\end{array}$ & 368.147 & $\mathrm{C}_{18} \mathrm{H}_{24} \mathrm{O}_{8}$ & MS \\
\hline 15 & 6.53 & $\begin{array}{l}\text { Dihydroxyphenyl)ethyl (1S,4aR,7aR)-1- } \\
(\beta \text {-D-glucopyranosyloxy)-4a-hydroxy-7- } \\
\text { methyl-5-oxo-1,4a,5,6,7,7ahexahydrocyclopenta[c]pyran-4- } \\
\text { carboxylate; } \Delta \text { Mass: } 274.1081 \mathrm{Da}]\end{array}$ & 252.061 & $\mathrm{C}_{8} \mathrm{H}_{8} \mathrm{~N}_{6} \mathrm{O}_{4}$ & MS \\
\hline 16 & 6.91 & $\begin{array}{l}\text { Unknown } \\
\text { [Similar to: 1-(Carboxymethyl)cyclohexanecarboxylic }\end{array}$ & 596.214 & $\mathrm{C}_{28} \mathrm{H}_{33} \mathrm{~N}_{6} \mathrm{O}_{7} \mathrm{P}$ & MS \\
\hline 17 & 6.91 & acid; $\Delta$ Mass: $-414.1466 \mathrm{Da}]$ & 600.236 & $\mathrm{C}_{21} \mathrm{H}_{43} \mathrm{~N}_{6} \mathrm{O}_{8} \mathrm{P}_{3}$ & MS \\
\hline 18 & 6.91 & $\begin{array}{l}\text { Similar to: 1-(Carboxymethyl)cyclohexanecarboxylic } \\
\text { Acid; } \Delta \text { Mass: }-208.0708 \mathrm{Da})\end{array}$ & 394.16 & $\mathrm{C}_{16} \mathrm{H}_{22} \mathrm{~N}_{6} \mathrm{O}_{6}$ & MS \\
\hline
\end{tabular}

**) Based on comparison of MS spectrum with database of National Institute of Standards and Technology (NIST) 
Table 2. Continued..

\begin{tabular}{|c|c|c|c|c|c|}
\hline $\begin{array}{c}\text { Peak } \\
\text { No }\end{array}$ & $\begin{array}{l}\text { Retention } \\
\text { Time (RT) in } \\
\text { minutes }\end{array}$ & Compounds & $\begin{array}{l}\text { Molecular } \\
\left.\text { Weight }^{* *}\right)\end{array}$ & $\begin{array}{l}\text { Molecular } \\
\left.\text { Formula }^{*}\right)\end{array}$ & $\begin{array}{c}\text { Reference } \\
\text { MS } \\
\text { Identification }^{* *} \text { ) }\end{array}$ \\
\hline 19 & 6.92 & $\begin{array}{l}\text { [Similar to: } 1- \\
\text { (Carboxymethyl)cyclohexanecarboxylic } \\
\text { acid; } \Delta \text { Mass: }-67.9870 \mathrm{Da}]\end{array}$ & 254.076 & $\mathrm{C}_{7} \mathrm{H}_{14} \mathrm{~N}_{2} \mathrm{O}_{8}$ & MS \\
\hline 20 & 6.99 & 1,3,3-trimethylindolino-6'-nitrobenzopyrylospiran & 322.131 & $\mathrm{C}_{19} \mathrm{H}_{18} \mathrm{~N}_{2} \mathrm{O}_{3}$ & MS \\
\hline 21 & 7.67 & 1,3-Bis(2,4-dimethoxyphenyl)-1,3-propanedione & 344.126 & $\mathrm{C}_{19} \mathrm{H}_{20} \mathrm{O}_{6}$ & MS \\
\hline 22 & 7.68 & $\begin{array}{l}\text { Rubone } \\
\text { [Similar to: [7-Hydroxy-1-(4-hydroxy-3, } \\
\text { 5-dimethoxyphenyl)-3-(hydroxymethyl)- }\end{array}$ & 374.136 & $\mathrm{C}_{20} \mathrm{H}_{22} \mathrm{O}_{7}$ & MS \\
\hline 23 & 8.03 & $\begin{array}{l}\text { 6,8-dimethoxy-1,2,3,4-tetrahydro-2- } \\
\text { naphthalenyl]methyl pentopyranoside; } \\
\Delta \text { Mass: -11.9636 Da] }\end{array}$ & 564.184 & $\mathrm{C}_{27} \mathrm{H}_{36} \mathrm{O}_{12}$ & MS \\
\hline 24 & 8.19 & $\begin{array}{l}\text { [Similar to: } 1- \\
\text { (Carboxymethyl)cyclohexanecarboxylic } \\
\text { acid; } \Delta \text { Mass: -208.0709 Da] }\end{array}$ & 394.16 & $\mathrm{C}_{16} \mathrm{H}_{22} \mathrm{~N}_{6} \mathrm{O}_{6}$ & MS \\
\hline 25 & 8.91 & $\begin{array}{l}\text { 4-Ethyl-3-oxa-5-thia-4- } \\
\text { boratricyclo[5.2.1.0 2,6 ]decane }\end{array}$ & 182.093 & $\mathrm{C}_{9} \mathrm{H}_{15} \mathrm{BOS}$ & MS \\
\hline 26 & 10.54 & Unknown & 524.263 & $\mathrm{C}_{29} \mathrm{H}_{32} \mathrm{~N}_{8} \mathrm{O}_{2}$ & - \\
\hline 27 & 10.96 & $\begin{array}{l}\text { Ethyl 5-methoxy-2-[(4- } \\
\text { methoxyphenoxy)methyl]-1- } \\
\text { benzofuran-3-carboxylate }\end{array}$ & 356.126 & $\mathrm{C}_{20} \mathrm{H}_{20} \mathrm{O}_{6}$ & MS \\
\hline 28 & 10.98 & $\begin{array}{l}\text { N-[2-(Adamantan-1-yloxy)ethyl]-3,5- } \\
\text { Dimethoxybenzamide }\end{array}$ & 359.209 & $\mathrm{C}_{21} \mathrm{H}_{29} \mathrm{NO}_{4}$ & MS \\
\hline 29 & 11 & $\begin{array}{l}(15 Z)-9,12,13-\text { Trihydroxy-15- } \\
\text { octadecenoic acid }\end{array}$ & 330.241 & $\mathrm{C}_{18} \mathrm{H}_{34} \mathrm{O}_{5}$ & MS \\
\hline 30 & 11.74 & $\begin{array}{l}\text { Ethyl 1,4-dioxaspiro[4.5]decane-6- } \\
\text { Carboxylate }\end{array}$ & 214.12 & $\mathrm{C}_{11} \mathrm{H}_{18} \mathrm{O}_{4}$ & MS \\
\hline 31 & 11.74 & $\begin{array}{l}\text { [Similar to: } 11(\mathrm{Z}), 14(\mathrm{Z}), 17(\mathrm{Z})- \\
\text { Eicosatrienoic acid; } \Delta \text { Mass: } 136.1262 \\
\text { Da] }\end{array}$ & 170.13 & - & MS \\
\hline 32 & 11.9 & $\begin{array}{l}\text { Isoindole-1,3-dione, 2-[2-(adamantan1-yloxy)ethyl]-5-methyl- } \\
\text { 3a,4,7,7atetrahydro }\end{array}$ & 343.214 & $\mathrm{C}_{21} \mathrm{H}_{29} \mathrm{NO}_{3}$ & MS \\
\hline 33 & 12.03 & 1-Allyl 2-dodecyl 1,2-pyrrolidinedicarboxylate & 367.272 & $\mathrm{C}_{21} \mathrm{H}_{37} \mathrm{NO}_{4}$ & MS \\
\hline 34 & 12.6 & Unknown & 508.268 & $\mathrm{C}_{29} \mathrm{H}_{32} \mathrm{~N}_{8} \mathrm{O}$ & MS \\
\hline 35 & 12.64 & Unknown & 547.279 & $\mathrm{C}_{31} \mathrm{H}_{33} \mathrm{~N}_{9} \mathrm{O}$ & MS \\
\hline 36 & 12.68 & $\begin{array}{l}\text { [Similar to: Cholic acid; } \Delta \text { Mass: }-0.9951 \\
\text { Da] }\end{array}$ & 409.283 & $\mathrm{C}_{24} \mathrm{H}_{35} \mathrm{~N}_{5} \mathrm{O}$ & MS \\
\hline 37 & 12.85 & 2-Nitro-1,3-bis(octyloxy)benzene & 379.272 & $\mathrm{C}_{22} \mathrm{H}_{37} \mathrm{NO}_{4}$ & MS \\
\hline 38 & 13.02 & Unknown & 427.293 & $\mathrm{C}_{24} \mathrm{H}_{37} \mathrm{~N}_{5} \mathrm{O}_{2}$ & - \\
\hline 39 & 13.04 & Unknown & 367.272 & $\mathrm{C}_{15} \mathrm{H}_{38} \mathrm{~N}_{5} \mathrm{O}_{3} \mathrm{P}$ & - \\
\hline 40 & 13.09 & Unknown & 397.282 & $\mathrm{C}_{23} \mathrm{H}_{35} \mathrm{~N}_{5} \mathrm{O}$ & - \\
\hline 41 & 13.19 & $\begin{array}{l}\text { (11alpha,13E,15S)-11,15-Dihydroxy-N- (2-hydroxyethyl)-9- } \\
\text { oxoprost-13-en-1- amide }\end{array}$ & 397.282 & $\mathrm{C}_{22} \mathrm{H}_{39} \mathrm{NO}_{5}$ & MS \\
\hline 42 & 13.55 & $\begin{array}{l}\text { [Similar to: 3-[2-(2,3-Dihydroxy-5,6,8atrimethyloctahydro- } \\
\text { 2Hspiro[naphthalene-1,2'-oxiran]-5- } \\
\text { yl)ethyl]-5-hydroxy-2(5H)-furanone; } \\
\Delta \text { Mass: -73.0889 Da] } \\
\text { [Similar to: } 20 \text {-Hydroxy- }\end{array}$ & 439.239 & $\mathrm{C}_{18} \mathrm{H}_{42} \mathrm{~N}_{5} \mathrm{O}_{5} \mathrm{P}$ & MS \\
\hline 43 & 13.74 & $\begin{array}{l}\text { (5Z,8Z,11Z,14Z)-eicosatetraenoic acid; } \\
\Delta \text { Mass: -135.0531 Da] }\end{array}$ & 455.288 & $\mathrm{C}_{25} \mathrm{H}_{37} \mathrm{~N}_{5} \mathrm{O}_{3}$ & MS \\
\hline 44 & 13.88 & $\begin{array}{l}(9 Z, 12 Z)-6,8-D i h y d r o x y-9,12- \\
\text { octadecadienoic acid }\end{array}$ & 312.23 & $\mathrm{C}_{18} \mathrm{H}_{32} \mathrm{O}_{4}$ & MS \\
\hline 45 & 14.09 & Unknown & 425.278 & $\mathrm{C}_{24} \mathrm{H}_{35} \mathrm{~N}_{5} \mathrm{O}_{2}$ & MS \\
\hline
\end{tabular}

**) Based on comparison of MS spectrum with database of National Institute of Standards and Technology (NIST) 
Table 2. Continued...

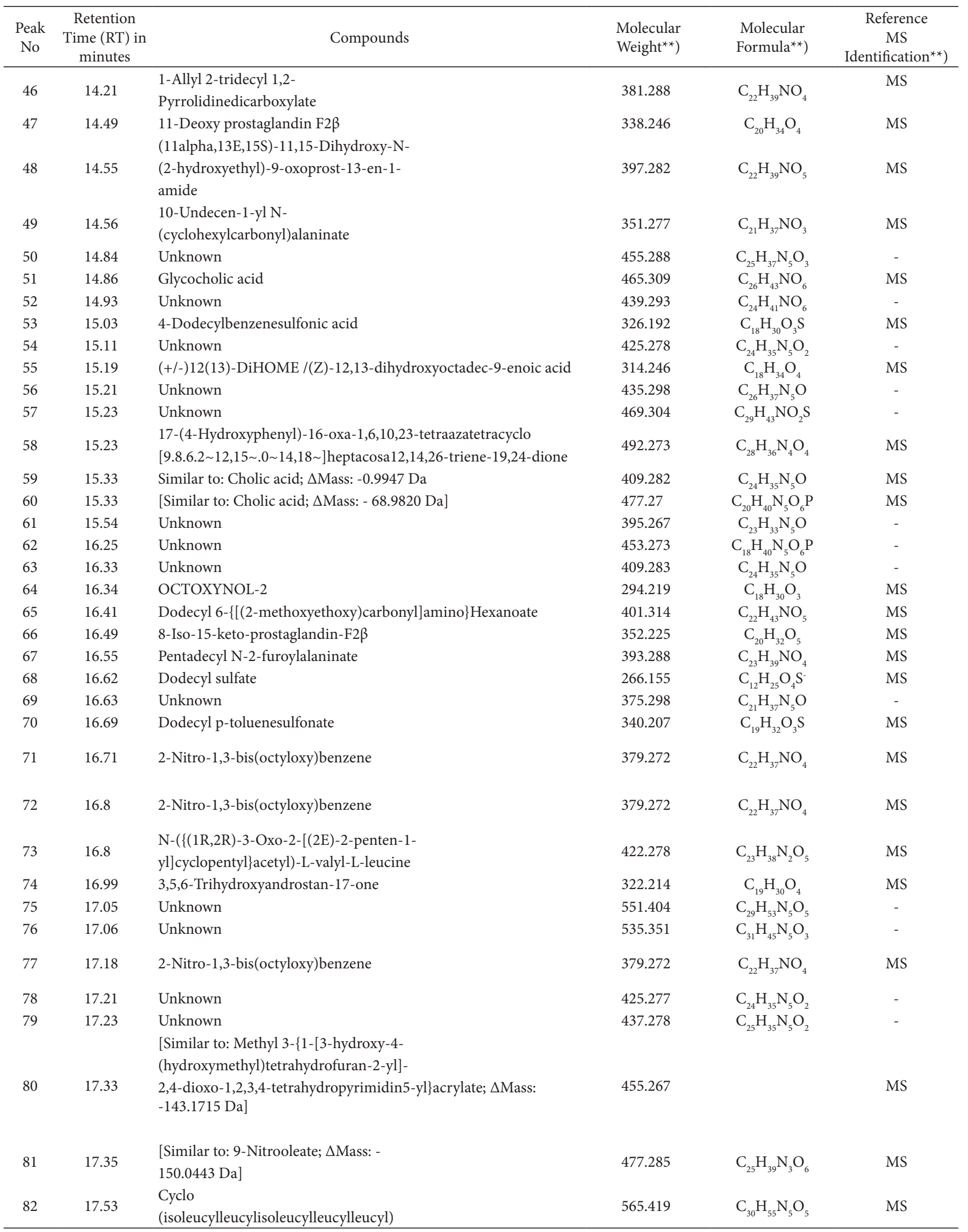

**) Based on comparison of MS spectrum with database of National Institute of Standards and Technology (NIST) 
Table 2. Continued...

\begin{tabular}{|c|c|c|c|c|c|}
\hline $\begin{array}{l}\text { Peak } \\
\text { No }\end{array}$ & $\begin{array}{l}\text { Retention } \\
\text { Time (RT) in } \\
\text { minutes }\end{array}$ & Compounds & $\begin{array}{l}\text { Molecular } \\
\text { Weight }^{* *} \text { ) }\end{array}$ & $\begin{array}{l}\text { Molecular } \\
\left.\text { Formula }^{\star *}\right)\end{array}$ & $\begin{array}{c}\text { Reference } \\
\text { MS } \\
\text { Identification }^{\star *} \text { ) }\end{array}$ \\
\hline 83 & 17.6 & DPPH & 395.086 & $\mathrm{C}_{18} \mathrm{H}_{12} \mathrm{~N}_{5} \mathrm{O}_{6}$ & MS \\
\hline 84 & 17.62 & Unknown & 596.296 & $\mathrm{C}_{28} \mathrm{H}_{45} \mathrm{~N}_{4} \mathrm{O}_{8} \mathrm{P}$ & - \\
\hline 85 & 17.63 & $\begin{array}{l}\text { [Similar to: 9-Nitrooleate; } \Delta \text { Mass: - } \\
150.0443 \mathrm{Da} \text { ] }\end{array}$ & 477.285 & $\mathrm{C}_{25} \mathrm{H}_{39} \mathrm{~N}_{3} \mathrm{O}_{6}$ & MS \\
\hline 86 & 17.64 & $\begin{array}{l}\text { [Similar to: 9-Nitrooleate; } \Delta \text { Mass: - } \\
\text { 178.0757 Da] }\end{array}$ & 505.317 & $\mathrm{C}_{21} \mathrm{H}_{44} \mathrm{~N}_{7} \mathrm{O}_{5} \mathrm{P}$ & MS \\
\hline 88 & 17.79 & Phenyl laurate & 276.209 & $\mathrm{C}_{18} \mathrm{H}_{28} \mathrm{O}_{2}$ & MS \\
\hline 89 & 17.81 & 4-Undecylbenzenesulfonic acid & 312.176 & $\mathrm{C}_{17} \mathrm{H}_{28} \mathrm{O}_{3} \mathrm{~S}$ & MS \\
\hline 90 & 17.9 & Unknown & 679.412 & $\mathrm{C}_{32} \mathrm{H}_{63} \mathrm{~N}_{3} \mathrm{O}_{8} \mathrm{P}_{2}$ & - \\
\hline 91 & 17.91 & Unknown & 601.397 & $\mathrm{C}_{30} \mathrm{H}_{56} \mathrm{ClN}_{5} \mathrm{O}_{5}$ & - \\
\hline 92 & 18.04 & $\begin{array}{l}\text { Methyl N- } \\
\text { [(benzyloxy)carbonyl] } \\
\text { leucylleucylleucinate }\end{array}$ & 505.317 & $\mathrm{C}_{27} \mathrm{H}_{43} \mathrm{~N}_{3} \mathrm{O}_{6}$ & MS \\
\hline 93 & 18.17 & $\begin{array}{l}\text { 5-(4-Carboxy-3-methylbutyl)-1,4adimethyl-6- } \\
\text { methylenedecahydro-1- } \\
\text { naphthalenecarboxylic acid }\end{array}$ & 336.23 & $\mathrm{C}_{20} \mathrm{H}_{32} \mathrm{O}_{4}$ & MS \\
\hline 94 & 18.31 & OCTOXYNOL-2 & 294.219 & $\mathrm{C}_{18} \mathrm{H}_{30} \mathrm{O}_{3}$ & MS \\
\hline 95 & 18.34 & 13(S)-Hydroperoxylinolenic acid & 246.199 & $\mathrm{C}_{18} \mathrm{H}_{30} \mathrm{O}_{4}$ & MS \\
\hline 96 & 18.42 & Unknown & 345.288 & $\mathrm{C}_{19} \mathrm{H}_{39} \mathrm{NO}_{4}$ & - \\
\hline 97 & 18.53 & Unknown & 407.267 & $\mathrm{C}_{17} \mathrm{H}_{38} \mathrm{~N}_{5} \mathrm{O}_{4} \mathrm{P}$ & - \\
\hline 98 & 18.54 & $\begin{array}{l}\text { [Similar to: Phosphatidylinositol-1,2- } \\
\text { dipalmitoyl; } \Delta \text { Mass: } 238.2296 \mathrm{Da} \text { ] }\end{array}$ & 572.296 & $\mathrm{C}_{27} \mathrm{H}_{41} \mathrm{~N}_{8} \mathrm{O}_{4} \mathrm{P}$ & MS \\
\hline 99 & 18.7 & HEXADECYL 4-NITROPHENYL ETHER & 363.277 & $\mathrm{C}_{22} \mathrm{H}_{37} \mathrm{NO}_{3}$ & MS \\
\hline 100 & 18.72 & 17-EPIOXANDROLONE & 306.219 & $\mathrm{C}_{19} \mathrm{H}_{30} \mathrm{O}_{3}$ & MS \\
\hline 101 & 18.76 & Unknown & 533.335 & $\mathrm{C}_{31} \mathrm{H}_{43} \mathrm{~N}_{5} \mathrm{O}_{3}$ & - \\
\hline 102 & 18.94 & 16,17-Dihydroxykauran-18-oic acid & 290.225 & $\mathrm{C}_{20} \mathrm{H}_{32} \mathrm{O}_{4}$ & MS \\
\hline 103 & 18.99 & $\begin{array}{l}\text { [Similar to: Phosphatidylinositol-1,2- } \\
\text { dipalmitoyl; } \Delta \text { Mass: } 329.2087 \mathrm{Da} \text { ] }\end{array}$ & 481.317 & $\mathrm{C}_{24} \mathrm{H}_{44} \mathrm{~N}_{5} \mathrm{O}_{3} \mathrm{P}$ & MS \\
\hline 104 & 19.05 & $\begin{array}{l}\text { [Similar to: Sorbitan monooleate; } \\
\Delta \text { Mass: }-50.9877 \mathrm{Da}]\end{array}$ & 479.302 & $\mathrm{C}_{18} \mathrm{H}_{43} \mathrm{~N}_{9} \mathrm{O}_{2} \mathrm{P}_{2}$ & MS \\
\hline 105 & 19.26 & 4-Dodecylbenzenesulfonic acid & 326.192 & $\mathrm{C}_{18} \mathrm{H}_{30} \mathrm{O}_{3} \mathrm{~S}$ & MS \\
\hline 106 & 19.26 & Unknown & 598.312 & $\mathrm{C}_{28} \mathrm{H}_{47} \mathrm{~N}_{4} \mathrm{O}_{8} \mathrm{P}$ & - \\
\hline 107 & 19.38 & Unknown & 563.346 & $\mathrm{C}_{32} \mathrm{H}_{45} \mathrm{~N}_{5} \mathrm{O}_{4}$ & - \\
\hline 108 & 19.51 & $\begin{array}{l}\text { Laurophenone } \\
1-\{[(2-\end{array}$ & 260.214 & $\mathrm{C}_{18} \mathrm{H}_{28} \mathrm{O}$ & MS \\
\hline 109 & 19.58 & $\begin{array}{l}\text { Aminoethoxy)(hydroxy)phosphoryl]oxy\} } \\
\text {-3-hydroxy-2-propanyl (4Z)-4- } \\
\text { icosenoate }\end{array}$ & 507.333 & $\mathrm{C}_{25} \mathrm{H}_{50} \mathrm{NO}_{7} \mathrm{P}$ & MS \\
\hline
\end{tabular}

${ }^{* *}$ ) Based on comparison of MS spectrum with database of National Institute of Standards and Technology (NIST)

Lariciresinol 4-O-glucoside is a lignan, a type of phenylpropanoids. In food, it is found in sesame seeds, flax and Brassica vegetables (Tchoumtchoua et al., 2019). It is also found in the bark and wood of white fir (Abies alba). The lignans are a large group of polyphenols found in plants and one of the major classes of phytoestrogens. It also acts as antioxidants and has potential anti-inflammatory activity (Korkina et al., 2011).

Phosphocholine is the phosphate of choline; and the parent compound of the phosphocholine family. It has a role as an epitope, a hapten, a human metabolite, a mouse metabolite, and an allergen. It is the conjugate acid of a choline phosphate (1-). Phosphocoline was also found in other fruits such as strawberry and Longan (Antunes et al., 2019; Wang et al., 2020).
There is a compound that is identified similar to Disperse red 17. Disperse dyes are a class of water-insoluble dyes that penetrate synthetic fibers and are held in place by physical forces without forming chemical bonds (Al-Etaibi \& El-Apasery, 2019). It is used as a colorant, commonly in hair dyeing agents and the textile industry. Disperse red-17 is a red dye that has red powder, soluble in ethanol and acetone. Other than that, 1,3,3-trimethylindolino6'-nitrobenzopyrylospiran also detected in this research. It is a photochromic compound. It can change their molecular structures by reversible process upon irradiation of lights of two different wavelengths. Several photochromic compounds were known to have the stability of heat (Malinauskiene et al., 2013). 
These results indicate the potential of Buni fruit as a source of good quality natural colorant.

Rubone was also detected in this research. This compound was also found in another plant such as Malva verticillata and Myrica nagi fruit (Bao et al., 2018; Patel \& Prashar, 2020) Several studies have shown that it has vigorous anticancer activity. Rubone and paclitaxel (PTX) combination therapy retarded cancer cell growth, migration and cancer stem-like cells (CSC) population growth (Xiao \& Chen, 2015).

This study also found that several compounds belong to the steroid group. 11-Deoxy prostaglandin F2 $\beta$ is an analog of PGF2. 8-Iso-15-keto-prostaglandin-F2 $\beta$ is an isomer of PGF2 $\alpha$ of non-enzymatic origin. These results indicate the potential of Antidesma bunius fruit to be used as a functional food.

\section{Conclusions}

Based on the study results, it can be concluded that the volatile components present in Antidesma bunius extract identified by GC-MS method as many as fifty compounds. Among these components, 5-hydroxymethylfurfural (5-HMF) was the one found in the greatest concentration. Also, there are ten predominant components and several compounds that can be used as flavoring agents. Antidesma bunius also contained several organic acids, long-chain fatty acids, and photochromic compounds. The compounds detected in this study may have biological activities which showed the great potential of Buni fruit to be applied in several aspects such as functional food, food processing and medicine.

\section{Acknowledgements}

This research was supported by the Indonesia Endowment Fund for Education (LPDP).

\section{References}

Al-Etaibi, A. M., \& El-Apasery, M. A. (2019). Dyeing performance of disperse dyes on polyester fabrics using eco-friendly carrier and their antioxidant and anticancer activities. International Journal of Environmental Research and Public Health, 16(23), 4603. http:// dx.doi.org/10.3390/ijerph16234603. PMid:31757022.

Antunes, A. C. N., Acunha, T. dos S., Perin, E. C., Rombaldi, C. V., Galli, V., \& Chaves, F. C. (2019). Untargeted metabolomics of strawberry (Fragaria $\mathrm{x}$ ananassa 'Camarosa') fruit from plants grown under osmotic stress conditions. Journal of the Science of Food and Agriculture, 99(15), 6973-6980. http://dx.doi.org/10.1002/jsfa.9986. PMid:31414485.

Aprea, E., Biasioli, F., \& Gasperi, F. (2015). Volatile compounds of raspberry fruit: from analytical methods to biological role and sensory impact. Molecules, 20(2), 2445-2474. https://doi.org/10.3390/ molecules20022445.

Balamurugan, R., Stalin, A., Aravinthan, A., \& Kim, J. H. (2015). $\gamma$-sitosterol a potent hypolipidemic agent: in silico docking analysis. Medicinal Chemistry Research, 24(1), 124-130. http://dx.doi. org/10.1007/s00044-014-1075-0.

Bao, L., Bao, X., Li, P., Wang, X., \& Ao, W. (2018). Chemical profiling of Malva verticillata L. by UPLC-Q-TOF-MSE and their antioxidant activity in vitro. Journal of Pharmaceutical and Biomedical Analysis,
150, 420-426. http://dx.doi.org/10.1016/j.jpba.2017.12.044. PMid:29289893.

Čechovská, L., Cejpek, K., Konečný, M., \& Velíšek, J. (2011). On the role of 2,3-dihydro-3,5-dihydroxy-6-methyl-(4H)-pyran-4-one in antioxidant capacity of prunes. European Food Research and Technology, 233(3), 367-376. http://dx.doi.org/10.1007/s00217-011-1527-4.

Chanukya, B. S., Prakash, M., \& Rastogi, N. K. (2017). Extraction of citric acid from fruit juices using supported liquid membrane. Journal of Food Processing and Preservation, 41(1), 1-10. http:// dx.doi.org/10.1111/jfpp.12790.

Chowtivannakul, P., Srichaikul, B., \& Talubmook, C. (2016). Hypoglycemic and hypolipidemic effects of seed extract from Antidesma bunius (L.) spreng in streptozotocin-induced diabetic rats. Pakistan Journal of Biological Sciences, 19(5), 211-218. http://dx.doi.org/10.3923/ pjbs.2016.211.218. PMid:29023025.

Deryabin, D. G., \& Tolmacheva, A. A. (2015). Antibacterial and antiquorum sensing molecular composition derived from quercus cortex (Oak bark) extract. Molecules (Basel, Switzerland), 20(9), 17093-17108. http://dx.doi.org/10.3390/molecules200917093. PMid:26393551.

Dieryckx, C., Gaudin, V., Dupuy, J. W., Bonneu, M., Girard, V., \& Job, D. (2015). Beyond plant defense: insights on the potential of salicylic and methylsalicylic acid to contain growth of the phytopathogen Botrytis cinerea. Frontiers in plant science, 6, 859. http://dx.doi. org/10.3389/fpls.2015.00859. PMid:26528317.

Ferlinahayati, F., Apriyaty, R., \& Eliza, E. (2016). Fatty acid and Alkenil Glycoside from the fruits of Mengkudu (Morinda citrifolia Linn). Indonesian Journal of Fundamental and Applied Chemistry, 1(2), 52-54. http://dx.doi.org/10.24845/ijfac.v1.i2.52.

Frisvad, J. C., Møller, L. L. H., Larsen, T. O., Kumar, R., \& Arnau, J. (2018). Safety of the fungal workhorses of industrial biotechnology: update on the mycotoxin and secondary metabolite potential of Aspergillus niger, Aspergillus oryzae, and Trichoderma reesei. Applied Microbiology and Biotechnology, 102(22), 9481-9515. http://dx.doi. org/10.1007/s00253-018-9354-1. PMid:30293194.

Gomes, F. N. D. C., Pereira, L. R., Ribeiro, N. F. P., \& Souza, M. M. V. M. (2015). Production of 5-hydroxymethylfurfural (HMF) via fructose dehydration: Effect of solvent and salting-out. Brazilian Journal of Chemical Engineering, 32(1), 119-126. http://dx.doi. org/10.1590/0104-6632.20150321s00002914.

Hardinasinta, G., Mursalim, M., Muhidong, J., \& Salengke, S. (2020). Determination of some chemical compounds of bignay (Antidesma bunius) fruit juice. Food Science and Technology, In press. http:// dx.doi.org/10.1590/fst.27720.

Hu, B., Lu, Q., Jiang, X. Y., Dong, X. C., Cui, M. S., Dong, C. Q., \& Yang, Y. P. (2017). Insight into the formation of Anhydrosugars in glucose pyrolysis: a joint computational and experimental investigation. Energy \& Fuels, 31(8), 8291-8299. http://dx.doi.org/10.1021/acs. energyfuels.7b01250.

Jorjong, S., Butkhup, L., \& Samappito, S. (2015). Phytochemicals and antioxidant capacities of Mao-Luang (Antidesma bunius L.) cultivars from Northeastern Thailand. Food Chemistry, 181, 248-255. http:// dx.doi.org/10.1016/j.foodchem.2015.02.093. PMid:25794747.

Jung, Y. Y., Hwang, S. T., Sethi, G., Fan, L., Arfuso, F., \& Ahn, K. S. (2018). Potential anti-inflammatory and anti-cancer properties of farnesol. Molecules (Basel, Switzerland), 23(11), 1-15. http://dx.doi. org/10.3390/molecules23112827. PMid:30384444.

Korkina, L., Kostyuk, V., De Luca, C., \& Pastore, S. (2011). Plant Phenylpropanoids as Emerging Anti-Inflammatory Agents. MiniReviews in Medicinal Chemistry, 11(10), 823-835. http://dx.doi. org/10.2174/138955711796575489. PMid:21762105. 
Kromidas, L., Perrier, E., Flanagan, J., Rivero, R., \& Bonnet, I. (2006). Release of antimicrobial actives from microcapsules by the action of axillary bacteria. International Journal of Cosmetic Science, 28(2), 103-108. http://dx.doi.org/10.1111/j.1467-2494.2006.00283.x. PMid:18492144.

Lizardo, R. C. M., Mabesa, L. B., Dizon, E. I., \& Aquino, N. A. (2015). Functional and antimicrobial properties of bignay [Antidesma bunius (L.) Spreng.] extract and its potential as natural preservative in a baked product. International Food Research Journal, 22(1), 88-95.

Malinauskiene, L., Bruze, M., Ryberg, K., Zimerson, E., \& Isaksson, M. (2013). Contact allergy from disperse dyes in textiles-a review. Contact Dermatitis, 68(2), 65-75. http://dx.doi.org/10.1111/cod.12001. PMid:23289879.

Miyamoto, J., Hasegawa, S., Kasubuchi, M., Ichimura, A., Nakajima, A., \& Kimura, I. (2016). Nutritional signaling via free fatty acid receptors. International Journal of Molecular Sciences, 17(4), 450. http://dx.doi.org/10.3390/ijms17040450. PMid:27023530.

Narayanan, A., Baskaran, S. A., Amalaradjou, M. A. R., \& Venkitanarayanan, K. (2015). Anticarcinogenic properties of medium chain fatty acids on human colorectal, skin and breast cancer cells in vitro. International Journal of Molecular Sciences, 16(3), 5014-5027. http:// dx.doi.org/10.3390/ijms16035014. PMid:25749477.

National Center for Biotechnology Information. (2020). PubChem. Rockville, Bethesda: NHI. Retrieved from https://pubchem.ncbi. nlm.nih.gov

Nikolov, P. Y., \& Yaylayan, V. A. (2011). Thermal decomposition of 5-(hydroxymethyl)-2-furaldehyde (HMF) and its further transformations in the presence of glycine. Journal of Agricultural and Food Chemistry, 59(18), 10104-10113. http://dx.doi.org/10.1021/ jf202470u. PMid:21838257.

Patel, N. J., \& Prashar, Y. (2020). High-performance thin-layer chromatography analysis of gallic acid and other phytoconstituents of methanolic extracts of Myrica nagi fruit. Phcog Res, 12(2), 95-101. http://dx.doi.org/10.4103/pr.pr_104_19.

Preininger, M., Gimelfarb, L., Li, H.-C., Dias, B. E., Fahmy, F., \& White, J. (2008). Dihydromaltol (2,3-dihydro-5-hydroxy-6-methyl-4H-pyran4-one): identification as a potent aroma compound in Ryazhenka kefir and sensory evaluation. Expression of Multidisciplinary Flavour Science, 3, 406-410. Retrieved from https://home.zhaw.ch/yere/pdf/ Teil102\%20-\%20Expression\%20of\%20Multidisciplinary.pdf
Puspitasari, E., \& Ulfa, E. U. (2009). Uji Sitotoksisitas Ekstrak Metanol Buah Buni (Antidesma bunius (L) Spreng) terhadap Sel Hela Cytotoxicity Effect of Methanolic Extract of Buni 's Fruits (Antidesma bunius (L) Spreng) against Hela Cells. Jurnal Ilmu Dasar, 10(2), 181-185.

Royal Society of Chemistry. (2020). ChemSpider. London: Royal Society of Chemistry. Retrieved from http://www.chemspider.com

Saeed, A., Kauser, S., \& Iqbal, M. (2018). Nutrient, mineral, antioxidant, and anthocyanin profiles of different cultivars of syzygium cumini (Jamun) at different stages of fruit maturation. Pakistan Journal of Botany, 50(5), 1791-1804.

Saini, R. K., \& Keum, Y. S. (2017). Characterization of nutritionally important phytoconstituents in bitter melon (Momordica charantia L.) fruits by HPLC-DAD and GC-MS. Journal of Food Measurement and Characterization, 11(1), 119-125. http://dx.doi.org/10.1007/ s11694-016-9378-0.

Sangeetha, J., \& Vijayalakshmi, K. (2011). Determination of bioactive components of ethyl acetate fraction of Punica granatum Rind Extract. International Journal of Pharmaceutical Sciences and Drug Research, 3(2), 116-122.

Tchoumtchoua, J., Mathiron, D., Pontarin, N., Gagneul, D., van Bohemen, A. I., Otogo N’nang, E., Mesnard, F., Petit, E., Fontaine, J. X., Molinié, R., \& Quéro, A. (2019). Phenolic profiling of flax highlights contrasting patterns in winter and spring varieties. Molecules (Basel, Switzerland), 24(23), 1-14. http://dx.doi.org/10.3390/ molecules24234303. PMid:31779076.

Wang, J., Guo, D., Han, D., Pan, X., \& Li, J. (2020). A comprehensive insight into the metabolic landscape of fruit pulp, peel, and seed in two longan (Dimocarpus longan Lour.) varieties. International Journal of Food Properties, 23(1), 1527-1539. http://dx.doi.org/10. 1080/10942912.2020.1815767.

Wankasi, D., \& Tarawou, T. (2013). Furfural production from the peels of ripe english mango (Mangnifera indica) fruits by acid catalyzed hydrolysis. Agriculture and Biology Journal of North America, 4(3), 216-220. http://dx.doi.org/10.5251/abjna.2013.4.3.216.220.

Xiao, Z., \& Chen, Y. (2015). Small molecule targeting miR-34a for cancer therapy. Molecular \& Cellular Oncology, 2(1), e977160. http://dx.doi. org/10.4161/23723556.2014.977160. PMid:27308410.

Yuan, G. F., Chen, X. E., \& Li, D. (2014). Conjugated linolenic acids and their bioactivities: A review. Food \& Function, 5(7), 1360-1368. http://dx.doi.org/10.1039/c4fo00037d. PMid:24760201. 